Wolf-Rayet Phenomena in Massive Stars and Starburst Galaxies

Proceedings IAU Symposium No. 193, (C)1999 IAU

K.A. van der Hucht, G. Koenigsberger \& P.R.J. Eenens, eds.

\title{
Starburst galaxy NGC 7673: a minor merger remnant
}

\author{
Nicole L. Homeier and John S. Gallagher \\ Astronomy Department, University of Wisconsin-Madison, \\ Madison, WI 53706, USA
}

\begin{abstract}
We investigate the kinematics of the luminous starburst galaxy NGC $7673=$ Mrk 325, a nearby example of a 'clumpy irregular galaxy'. Images with the WIYN $3.5 \mathrm{~m}$ telescope reveal outer wisps associated with mergers, and we suggest NGC 7673 is the survivor of a minor merger. DensePak spectroscopy of the $\mathrm{H} \alpha$ kinematics shows that the $\mathrm{H}$ II regions are confined to a rotating disk. The $\mathrm{H} \alpha$ emission line velocity field indicates that although there is widespread star formation across the inner disk, it is still rotating smoothly. The $\mathrm{H} \alpha$ emission profiles over the brightest regions of the galaxy have two components: narrow, $\mathrm{FWHM} \approx 50 \mathrm{~km} \mathrm{~s}^{-1}$ lines, and broad, FWHM $\approx 150 \mathrm{~km} \mathrm{~s}^{-1}$ lines. The width of the narrow lines is likely due to a combination of averaging effects, stellar winds and SNRs, and possibly gravitational motions within the disk. The origin of the broad component, which eclipses the narrow component in flux over much of the galaxy's inner regions, is still unclear. We may be looking at turbulent gas encased in bubbles carved out by massive stars and their descendants, or possibly some localized outflows from the disk.
\end{abstract}

Early studies of NGC 7673 characterized it as a clumpy, irregular galaxy, a description it earned from its giant star-forming regions embedded in a diffuse halo (Markarian \& Lipovetski 1971; Börngen \& Kalloglyan 1975; Casini \& Heidmann 1976; Coupinot et al. 1982). A fairly uniform velocity was measured from emission line kinematics by Duflot-Augarde \& Alloin (1982), and they postulated that the cause of NGC 7673's peculiarities is a tidal interaction with the nearby NGC 7677. However, recent low resolution $\mathrm{HI}$ measurements have shown that the outer contours of HI flux around this galaxy are fairly smooth. There is a noticeable lack of classic interaction signatures, such a $\mathrm{HI}$ tidal tails, making the NGC 7677 explanation less palatable.

In this study we have combined a number of data sets to study both the nature of the starburst occuring in NGC 7673, and also the possible trigger. With the WIYN $3.5 \mathrm{~m}$ telescope, we have obtained $B, R$, and $\mathrm{H} \alpha$ imaging, and integral field spectroscopy with DensePak to measure the $\mathrm{H} \alpha$ kinematics. DensePak is a rectangular $\left(30^{\prime \prime} \times 45^{\prime \prime}\right)$ array with fiber diameters of $3^{\prime \prime}$ and fiber centers separated by $4^{\prime \prime}$.

We find the $\mathrm{H} \alpha$ radial velocities to be fairly constant, with only a $60 \mathrm{~km} \mathrm{~s}^{-1}$ gradient over the 6 by $9 \mathrm{kpc}$ region covered by DensePak. Such a shallow velocity gradient in combination with the narrow emission line component indicates that the $\mathrm{H}$ II regions are confined to a dynamically cold rotating disk that is nearly face-on. Over much of the galaxy, the $\mathrm{H} \alpha$ line profiles have broad wings. Deconvolution of these profiles into narrow and broad Gaussian components gives a good fit, with mean FWHMs of 56 and $149 \mathrm{~km} \mathrm{~s}^{-1}$, respectively. Most of the 
broad components are slightly offset to the blue of the narrow components, but never by more than $10 \mathrm{~km} \mathrm{~s}^{-1}$.

Large, luminous clumps identified in the $B$ and $R$-band images are also bright in the $\mathrm{H} \alpha$ image, identifying them as giant star-forming regions. There are other interesting features in the broad band images which are not seen in the $\mathrm{H} \alpha$ image. Surrounding the bright central disk of NGC 7673 are wispy, lowsurface brightness features, including ripples and arcs usually associated with merger candidate E and S0 galaxies. These structures are present in both the $B$ and $R$ images, but not $\mathrm{H} \alpha$, indicating that they are not sites of recent starformation, but are composed of older stars.

Although the creation mechanism of ripples is still the subject of debate, observational evidence shows that these structures are associated with mergers(Grothues \& Schmidt-Kaler 1991; Hunter et al. 1994; Mulder \& van Driel 1996; Schweizer \& Seitzer 1988). The detections of ripples around NGC 7673 is strong evidence for a merger as the starburst trigger. The existence of a uniformly rotating disk and smooth $\mathrm{H}$ I morphology indicate that any interloper must have been much smaller than the accreting spiral galaxy, probably $<10 \%$ of the disk mass (Hernquist \& Mihos 1995), making this a candidate minor merger.

In summary, a minor merger appears to have triggered intense star formation across the inner $8 \mathrm{kpc}$ of the disk of NGC 7673, rendering it morphologically peculiar. Yet despite this galaxy's disturbed optical appearance, it has a regular velocity field, indicating it retains a dynamically cold disk.

\section{References}

Börngen, F., Kalloglyan, A. 1975, ApJ 92, 567

Casini, C., Heidmann, J. 1976, A\&A 47, 371

Coupinot, G., Hecquet, J., Heidmann, J. 1982, MNRAS 199, 451

Duflot-Augarde, R., Alloin, D. 1982 A\&A 112, 257

Grothues, H., Schmidt-Kaler, Th. 1991, A\&A 242, 357

Herquist, L., Mihos, J. 1995, ApJ 448, 41

Hunter, D., van Woerden, H., Gallagher, J. 1994, ApJS 91, 79

Markarian, B., Lipovetski, V. 1971, Astrofisika 7, 511

Mulder, P., van Driel, W. 1996, A\&A 309, 403

Nordgren, T., Chengalur, J., Salpeter, E., Terzian, Y. 1997, AJ 114, 77 